\title{
Gender differences in variables associated with dipeptidyl peptidase 4 genetic polymorphisms in coronary artery disease
}

\author{
*Shih-Min Chiang1,B,C,E, ${ }^{*} K_{w}$ o-Chang Ueng 2,3,A,B,E,F, Yi-Sun Yang 2,3,A,D,F \\ ${ }^{1}$ Institute of Medicine, Chung-Shan Medical University, Taichung, Taiwan \\ 2 School of Medicine, Chung-Shan Medical University, Taichung, Taiwan \\ ${ }^{3}$ Department of Internal Medicine, Chung-Shan Medical University Hospital, Taichung, Taiwan \\ A - research concept and design; B - collection and/or assembly of data; C - data analysis and interpretation; \\ $D$ - writing the article; $E$ - critical revision of the article; $F$ - final approval of the article
}

\section{Address for correspondence}

Yi-Sun Yang

E-mail: submission119@gmail.com

\section{Funding sources}

This work was supported by grants from the Chung Shan Medical University Hospital (grant No. CSH-2014-C-008).

Conflict of interest

None declared

* Shih-Min Chiang and Kwo-Chang Ueng shared equal contribution as first author.

\section{Received on March 16, 2020}

Reviewed on July 4, 2020

Accepted on August 11, 2020

Published online on 0ctober 8, 2020

\begin{abstract}
Background. In recent years, considerable effort has been devoted to identifying genes that contribute to the risk of coronary artery disease (CAD). Genetic factors can be used to identify individuals who have additional genetic risks. Genetic variations might contribute to cardiovascular disease differentially in men and women. Dipeptidyl peptidase-4 (DPP-4) may be involved in the development of atherosclerotic diseases.

Objectives. To examine the associations between genetic variations of DPP-4 in men and women with CAD.

Material and methods. In this case-control study, blood samples of patients with angiographically documented CAD and of those without CAD were collected. We focused on the DPP-4 gene (rs7608798 and rs3788979 polymorphisms) to assess the association of single nucleotide polymorphisms (SNPS) and the risk of CAD.

Results. We identified 1 SNP (rs3788979) that was significantly related to angiographic CAD in women (odds ratio $(O R)=2.437 ; p=0.019)$. Moreover, the SNP (rs7608798) seemed to have a protective effect $(O R=0.291$; $p=0.032$. We did not find an association between CAD risk factors and DPP-4 polymorphisms. Our study is the first to demonstrate that CAD pathogenesis is influenced by gender differences in polymorphisms in the DPP-4 gene.
\end{abstract}

Conclusions. This study provides new information on the association of DPP-4 polymorphisms with the risk of CAD in the Taiwanese population, especially in women. Further studies should be performed to verify this association.

Key words: women, coronary artery disease, DPP-4 polymorphisms

Cite as

Chiang SM, Ueng KC, Yang YS. Gender differences in variables associated with dipeptidyl peptidase 4 genetic polymorphisms in coronary artery disease. Adv Clin Exp Med. 2020;29(10):1181-1186. doi:10.17219/acem/126291

DOI

10.17219/acem/126291

Copyright

Copyright by Author(s)

This is an article distributed under the terms of the

Creative Commons Attribution 3.0 Unported (CC BY 3.0)

(https://creativecommons.org/licenses/by/3.0/) 


\section{Introduction}

The increasing prevalence of coronary artery disease (CAD) has become a worldwide health concern. It imposes a significant social and economic burden on society. ${ }^{1,2}$

Several risk factors may predispose an individual to $\mathrm{CAD}$, such as smoking, high blood pressure, high cholesterol, obesity, and diabetes. ${ }^{3}$ In recent years, considerable effort has been devoted to identifying genes that contribute to CAD risk. ${ }^{4,5}$ Genetic factors can be used to identify people who have additional genetic risks. This raises the possibility of screening the population to detect important susceptibility loci once disease screening or an intervention becomes available. An obvious requirement for such screening would be validation through large-scale trials of the benefits of such early detection and treatment. A combination of conventional and genetic risk factors may be optimal for identifying populations at risk.

Dipeptidyl peptidase 4 (DPP-4) is an intrinsic membrane glycoprotein and a serine exopeptidase that cleaves $\mathrm{X}$-proline dipeptides from the N-terminus of polypeptides. The activity of DPP- 4 has received considerable attention as a therapeutic target, and DPP-4 inhibitors that extend the insulinotropic effect of glucagon-like peptide-1 are now widely used as antidiabetic drugs. ${ }^{6}$

The DPP-4 is expressed on the surface of several cell types ${ }^{7}$ of a variety of tissues, including bone marrow, lung, spleen, pancreas, kidney, liver, intestinal, and epithelial cells. ${ }^{7}$ Because of its many enzymatic activities, DPP-4 is not only involved in the pathogenesis of diabetes mellitus and glucose metabolism, but it also participates in modulating the cardiovascular system and it may be involved in the development of atherosclerotic diseases. ${ }^{8-10}$ In vitro preclinical and clinical studies have shown that DPP-4 inhibitors may modulate atherosclerotic disease by reducing plasma lipids, suppressing inflammation and promoting vascular relaxation. However, the inhibition of DPP-4 may also exacerbate cardiovascular disease by enhancing sympathetic activation and angiogenesis. ${ }^{8}$

Three recent cardiovascular outcome trials of DPP-4 inhibitors showed that none of the DPP-4 inhibitors increased or decreased the predefined primary cardiovascular outcomes. ${ }^{11-13}$ However, the main concern in the subsequent discussion has been about the higher incidence of hospitalization for heart failure seen with saxagliptin. ${ }^{12}$ The question of why this ambiguous result in the cardiovascular outcome of DPP-4 inhibitors in human and animal studies may be explained by genetic polymorphism. Previous genetic studies revealed that polymorphisms in the defined DPP-4 loci are associated with serum lipid levels. ${ }^{14}$ A polymorphism in the DPP-4 gene in patients with known CAD may increase the risk of myocardial infarction (MI). It is associated with decreased plasma DPP-4 levels in patients with MI. ${ }^{15}$ Due to the diverse results, more comprehensive study of the DPP-4 gene polymorphism is needed. However, there is still limited data to confirm an association between $D P P-4$ gene variants and CAD in Asian populations, especially in gender-specific gene variants. In this study, we hypothesized that the polymorphisms of the DPP-4 gene might contribute differently in men and women. Therefore, we conducted a case-control study to examine the associations between single nucleotide polymorphisms (SNPs) in patients with or without CAD in a Taiwanese population.

\section{Material and methods}

This hospital-based case-control study recruited 607 patients who had undergone coronary angiography from the Chung Shan Medical University Hospital in Taichung, Taiwan. Two groups were identified for this study. For CAD group, a diagnosis of CAD was made for patients who had undergone coronary angiography and received percutaneous coronary angioplasty according to the recommendations for percutaneous coronary intervention. For the control (non-CAD) group, all participants were recruited from the same hospital; they had undergone coronary angiography, but the findings were negative for CAD, defined as coronary flow reserve is increased in blood flow in response to metabolic or pharmacological stimuli and a normal coronary angiogram without any suspected atherosclerosis. The patients' clinic characteristics were verified with a medical history review.

Diabetes mellitus was defined as having a fasting blood sugar level $\geq 126 \mathrm{mg} / \mathrm{dL}$ or taking any oral or injected antidiabetic drugs. Dyslipidemia was defined as having low-density-lipoprotein cholesterol (LDL-C) level $>100 \mathrm{mg} / \mathrm{dL}$ or taking any anti-lipid lowering drugs. Hypertension was defined as having a systolic blood pressure $(\mathrm{SBP})>130 \mathrm{~mm} \mathrm{Hg}$ and a diastolic blood pressure (DBP) $>80 \mathrm{~mm}$ Hg or taking any anti-hypertension drugs.

Whole-blood specimens collected from all patients were placed in tubes containing EDTA; they were immediately centrifuged and then stored at $-80^{\circ} \mathrm{C}$. The study protocols were approved by the institutional review of the Taichung Chung Shan Medical University Hospital. All methods were carried out in accordance with the approved guidelines and all participants provided written informed consent before participating in the study.

The allelic discrimination of the DPP-4 rs7608798 and rs3788979 polymorphisms was assessed using an ABI StpOne Real-Time PCR System (Applied Biosystems, Foster City, USA), SDS v. 3.0 software (Applied Biosystems) and the TaqMan ${ }^{\text {TM }}$ Genotyping assays. ${ }^{17-19}$ The final volume for each reaction was $5 \mu \mathrm{L}$, containing $2.5 \mu \mathrm{L}$ of TaqMan Genotyping Master Mix, $0.125 \mu \mathrm{L}$ of TaqMan probe mix and 10 ng of genomic DNA. The reaction conditions included an initial denaturation step at $95^{\circ} \mathrm{C}$ for $10 \mathrm{~min}$, followed by 40 cycles at $95^{\circ} \mathrm{C}$ for $15 \mathrm{~s}$ and $60^{\circ} \mathrm{C}$ for $1 \mathrm{~min}$.

We compared the differences in demographic characteristics between the CAD patients and the non-CAD patients 
using the $\chi^{2}$ test and Fisher's exact test. The odds ratios (ORs) with 95\% confidence intervals (95\% CIs) were estimated using logistic regression models between genotype frequencies and CAD risk, as well as clinical characteristics after controlling for other covariates. Values of $\mathrm{p}<0.05$ were considered statistically significant. The data was analyzed using SPSS statistical software v. 9.1, 2005 (SAS Institute Inc., Cary, USA).

\section{Results}

A total of 607 patients were recruited. Statistical analyses of the demographic characteristics of both the case group (391 patients with CAD) and the control group (216 patients without CAD) are shown in Table 1. Coronary artery disease was more common in men and in those with a history of hypertension and diabetes mellitus. Furthermore, gender, tobacco consumption and co-morbid conditions of hypertension or diabetes mellitus were significantly associated with CAD risk ( $\mathrm{p}<0.05$ for each). To decrease the possible interference of several co-morbid factors, the OR was adjusted to control for risk related to gender, tobacco use and co-morbidities in each comparison using multiple logistic regression models.

The distribution frequency of $D P P-4$ genotypes in both controls and CAD patients are presented in Table 2. The alleles with the highest distribution frequency were heterozygous A/G for rs7608798 and heterozygous G/A for rs3788979. There was no significant difference with respect to the rs7608798 and rs3788979 polymorphisms of DPP-4 between the controls and the patients with CAD.

The gender distribution frequencies are presented in Tables 3 and 4. Heterozygous G/A rs3788979 in DPP-4 was associated with CAD risk $(\mathrm{OR}=2.437 ; \mathrm{p}=0.019)$ only in the female population. In this group, homozygous G/G rs7608798 seemed to have a protective effect $(O R=0.291 ; p=0.032)$.

We further investigated the effects of the polymorphic genotypes of DPP-4 on the clinical status of CAD (Table 5). No apparent significant association between $D P P-4$ gene polymorphism and traditional CAD risk factors were found, except for elevated cardiac enzymes.

\section{Discussion}

Two SNPs in the DPP-4 gene were found to have an association with CAD risk in our study: one was associated with a decreased risk in women, while the other was associated with an increased risk. The rs7608798, an intron variant in the DPP-4 gene, was found to be associated with a decreased risk of CAD in the Chinese population for the first time. In contrast, rs3788979, also located in an intronic region of the $D P P-4$ gene, increased the risk of CAD and showed a strong association signal for CAD in the DPP-4 gene. This finding might seem to be partly consistent with previous observations of positive correlations between the rs3788979 SNP and the risk of MI in patients with atherosclerosis and of CAD in patients with diabetes. ${ }^{15}$ Our study extends the findings to those

Table 1. Demographic characteristics and clinical parameters for controls (non-CAD) and patients with CAD

\begin{tabular}{|c|c|c|c|}
\hline Parameter & $\begin{array}{l}\text { Control group } \\
\quad(n=216) \\
\text { mean } \pm S D\end{array}$ & $\begin{array}{l}\text { CAD group } \\
(n=391) \\
\text { mean } \pm S D\end{array}$ & $p$-value \\
\hline Age [years] & $66.43 \pm 12.17$ & $65.73 \pm 11.21$ & 0.474 \\
\hline BMI $\left[\mathrm{kg} / \mathrm{m}^{2}\right]$ & $25.05 \pm 3.74$ & $25.55 \pm 4.40$ & 0.159 \\
\hline Parameter & $n(\%)$ & n (\%) & $\mathrm{p}$-value \\
\hline Age $>65$ years & $126(58.3 \%)$ & $210(53.7 \%)$ & 0.272 \\
\hline Male gender & $130(60.2 \%)$ & $288(73.7 \%)$ & 0.001 \\
\hline Family history of CAD & $49(22.7 \%)$ & $82(21.0 \%)$ & 0.623 \\
\hline Tobacco consumption & $76(35.2 \%)$ & $171(43.7 \%)$ & 0.040 \\
\hline More than 3 risk factors of CAD & $101(46.8 \%)$ & $231(59.1 \%)$ & 0.004 \\
\hline Atrial fibrillation & $57(26.4 \%)$ & $39(10.0 \%)$ & $<0.001$ \\
\hline Hypertension & $134(62.0 \%)$ & $288(73.7 \%)$ & 0.003 \\
\hline Diabetes mellitus & $73(33.8 \%)$ & $165(42.2 \%)$ & 0.042 \\
\hline Dyslipidemia & $83(39.0 \%)$ & $158(40.6 \%)$ & 0.693 \\
\hline Recent $(<24 \mathrm{~h}$ ) severe angina & $126(58.3 \%)$ & $285(73.3 \%)$ & $<0.001$ \\
\hline Elevated cardiac markers & 81 (37.5\%) & $222(56.8 \%)$ & $<0.001$ \\
\hline Stroke & $27(13.4 \%)$ & $42(11.3 \%)$ & 0.445 \\
\hline Congestive heart failure & $73(35.3 \%)$ & $86(22.8 \%)$ & 0.001 \\
\hline
\end{tabular}

The data is presented as numbers and percentages with $x^{2}$ test/Fisher's exact test or means \pm standard deviation (SD) with independent two-sample t-test. CAD - coronary artery disease; $\mathrm{BMI}$ - body mass index; $\mathrm{p}<0.05$ is statistically significant. 
Table 2. Distribution frequency of DPP-4 genotypes in 216 controls (non-CAD) and 391 patients with CAD

\begin{tabular}{|c|c|c|c|c|}
\hline Genotype & $\begin{array}{l}\text { Control group } \\
\quad(n=216)\end{array}$ & $\begin{array}{l}\text { CAD group } \\
(n=391)\end{array}$ & Adjusted OR (95\% Cl) & $p$-value \\
\hline \multicolumn{5}{|c|}{$\begin{array}{c}\text { DPP-4 } \\
\text { rs7608798 }\end{array}$} \\
\hline AA & $85(39.4 \%)$ & $153(39.1 \%)$ & 1.00 & - \\
\hline$A G$ & 98 (45.4\%) & 195 (49.9\%) & $1.105(0.772-1.584)$ & 0.585 \\
\hline GG & $33(15.3 \%)$ & $43(11.0 \%)$ & $0.724(0.428-1.224)$ & 0.228 \\
\hline$A G+G G$ & $131(60.6 \%)$ & 238 (60.9\%) & $1.009(0.718-1.418)$ & 0.957 \\
\hline \multicolumn{5}{|c|}{$\begin{array}{c}\text { DPP-4 } \\
\text { rs3788979 }\end{array}$} \\
\hline GG & $61(28.2 \%)$ & $91(23.3 \%)$ & 1.00 & - \\
\hline GA & $106(49.1 \%)$ & $215(55.0 \%)$ & $1.360(0.912-2.027)$ & 0.131 \\
\hline AA & $49(22.7 \%)$ & $85(21.7 \%)$ & $1.163(0.721-1.876)$ & 0.536 \\
\hline
\end{tabular}

The odds ratios (OR) with their 95\% confidence intervals (95\% Cl) were estimated with logistic regression; CAD - coronary artery disease; $\mathrm{p}<0.05$ is statistically significant.

Table 3. DPP-4 genetic variation frequencies and CAD risk association among men

\begin{tabular}{|c|c|c|c|c|}
\hline Genotype & $\begin{array}{l}\text { Control group (non-CAD) } \\
\qquad(n=130)\end{array}$ & CAD group $(n=288)$ & OR $(95 \% \mathrm{Cl})$ & $\mathrm{p}$-value \\
\hline \multicolumn{5}{|c|}{$\begin{array}{l}\text { DPP-4 } \\
\text { rs7608798 }\end{array}$} \\
\hline$A A$ & $54(41.5 \%)$ & $112(38.9 \%)$ & 1.00 & - \\
\hline$A G$ & $56(43.1 \%)$ & $138(47.9 \%)$ & $1.188(0.758-1.862)$ & 0.452 \\
\hline GG & $20(15.4 \%)$ & $38(13.2 \%)$ & $0.916(0.487-1.722)$ & 0.786 \\
\hline$A G+G G$ & $76(58.5 \%)$ & $176(61.1 \%)$ & $1.117(0.732-1.702)$ & 0.608 \\
\hline \multicolumn{5}{|c|}{$\begin{array}{c}\text { DPP-4 } \\
\text { rs3788979 }\end{array}$} \\
\hline GG & $37(28.5 \%)$ & $75(26.0 \%)$ & 1.00 & - \\
\hline GA & $66(50.8 \%)$ & $150(52.1 \%)$ & $1.121(0.688-1.828)$ & 0.646 \\
\hline AA & $27(20.7 \%)$ & $63(21.9 \%)$ & $1.151(0.633-2.094)$ & 0.645 \\
\hline
\end{tabular}

The odds ratios (OR) with their 95\% confidence intervals ( $95 \% \mathrm{Cl}$ ) were estimated with logistic regression; CAD - coronary artery disease; $\mathrm{p}<0.05$ is statistically significant.

Table 4. DPP-4 genetic variation frequencies and CAD risk association among women

\begin{tabular}{|c|c|c|c|c|}
\hline Genotype & $\begin{array}{l}\text { Control group } \\
\qquad(n=86)\end{array}$ & $\begin{array}{l}\text { CAD group } \\
(n=103)\end{array}$ & OR $(95 \% \mathrm{Cl})$ & p-value \\
\hline \multicolumn{5}{|c|}{$\begin{array}{c}\text { DPP-4 } \\
\text { rs7608798 }\end{array}$} \\
\hline AA & $31(36.0 \%)$ & $41(39.8 \%)$ & 1.00 & - \\
\hline$A G$ & $42(48.8 \%)$ & $57(55.3 \%)$ & $1.026(0.556-1.895)$ & 0.934 \\
\hline GG & $13(15.2 \%)$ & $5(4.9 \%)$ & $0.291(0.094-0.902)$ & $0.032^{*}$ \\
\hline$A G+G G$ & $55(64.0 \%)$ & $62(60.2 \%)$ & $0.852(0.472-1.539)$ & 0.596 \\
\hline \multicolumn{5}{|c|}{$\begin{array}{c}\text { DPP-4 } \\
\text { rs3788979 }\end{array}$} \\
\hline GG & $24(27.9 \%)$ & $16(15.5 \%)$ & 1.00 & - \\
\hline GA & $40(46.5 \%)$ & $65(63.1 \%)$ & $2.437(1.157-5.135)$ & $0.019^{*}$ \\
\hline AA & $22(25.6 \%)$ & $22(21.4 \%)$ & $1.500(0.631-3.565)$ & 0.359 \\
\hline
\end{tabular}

Odds ratios (OR) and 95\% confidence intervals $(95 \% \mathrm{Cl})$ of female CAD patients associated with genotypic frequencies of DPP-4; CAD - coronary artery disease; ${ }^{*} p<0.05$ is statistically significant. 
Table 5. Comparison of demographics and co-morbidities between 2 genotypes of DPP-4 rs7608798 in the group of CAD patients

\begin{tabular}{|c|c|c|c|c|}
\hline Variable & $\begin{array}{c}\text { DPP-4 rs7608798 } \\
\text { AA } \\
(n=153)\end{array}$ & $\begin{array}{l}\text { DPP-4 rs7608798 } \\
A G+G G(n=238)\end{array}$ & OR $(95 \% \mathrm{Cl})$ & $\mathrm{p}$-value \\
\hline Atrial fibrillation & $15(9.8 \%)$ & $24(10.1 \%)$ & $1.032(0.523-2.036)$ & 0.928 \\
\hline More than 3 risk factors of CAD & $90(58.8 \%)$ & $141(59.2 \%)$ & $1.018(0.673-1.538)$ & 0.934 \\
\hline Age $>65$ years & $78(51.0 \%)$ & $132(55.5 \%)$ & $1.197(0.797-1.799)$ & 0.386 \\
\hline Family history of CAD & $25(16.3 \%)$ & $57(23.9 \%)$ & $1.612(0.957-2.717)$ & 0.071 \\
\hline Hypertension & $110(71.9 \%)$ & $178(74.8 \%)$ & $1.160(0.733-1.834)$ & 0.526 \\
\hline Diabetes mellitus & $68(44.4 \%)$ & $97(40.8 \%)$ & $0.860(0.570-1.296)$ & 0.471 \\
\hline Tobacco consumption & $65(42.5 \%)$ & $106(44.5 \%)$ & $1.087(0.722-1.638)$ & 0.689 \\
\hline Dyslipidemia & $64(42.4 \%)$ & $94(39.5 \%)$ & $0.887(0.586-1.343)$ & 0.572 \\
\hline Recent ( $<24$ h) severe angina & $114(75.0 \%)$ & $171(72.2 \%)$ & $0.864(0.543-1.374)$ & 0.536 \\
\hline Elevated cardiac markers & $97(63.4 \%)$ & $125(52.5 \%)$ & $0.639(0.421-0.968)$ & $0.034^{*}$ \\
\hline Stroke & $17(11.7 \%)$ & $25(11.0 \%)$ & $0.927(0.482-1.784)$ & 0.821 \\
\hline Congestive heart failure & $27(18.5 \%)$ & $59(25.5 \%)$ & $1.512(0.906-2.522)$ & 0.112 \\
\hline
\end{tabular}

The odds ratios (OR) with their 95\% confidence intervals $(95 \% \mathrm{Cl})$ were estimated with logistic regression; CAD - coronary artery disease; ${ }^{*} \mathrm{p}<0.05$ is statistically significant.

without diabetes, but we only found this positive correlation between the rs3788979 SNP and the risk of CAD in female patients.

In dominant inheritance mode, the carriers of genotype GA at the rs3788979 SNP had an increased risk of CAD in comparison with the carriers of AA after adjusting for other CAD risk factors.

Cardiovascular disease constitutes the leading cause of mortality, not only among men but also among women. ${ }^{16}$ Gender differences in the incidence of CAD have attracted the interest of many clinical researchers. Some gender differences have been documented in regard to traditional cardiovascular risk factors. Age, hypertension, total cholesterol, and LDL-C have a great influence on men, while menopause, systolic arterial hypertension, smoking, diabetes, and triglyceride and high-density-lipoprotein cholesterol (HDL-C) levels are the main factors in women. ${ }^{17}$ Genetic variations might not explain all of these disparities, but they could provide some further information on gender differences regarding the risk of cardiovascular disease.

Although the precise mechanisms that link DPP-4 and cardiovascular disease are not completely understood, various studies have suggested that DPP-4 inhibitors may also have cardiovascular protective effects. ${ }^{18-20}$ On the other hand, some animal studies have shown that DPP-4 deficiency may cause dyslipidemia, ${ }^{21}$ and another study revealed that elevated DPP-4 levels accelerate diet-related vascular aging and atherosclerosis. ${ }^{22}$

Moreover, a recent study reported that a genetic deficiency of DPP-4 improves cardiac function, ${ }^{23}$ whereas inhibition of DPP-4 induced cardiac hypertrophy and impaired cardiac function and reduced monocyte migration to atherosclerotic plaque in response to tumor necrosis factor $\alpha$ (TNF- $\alpha$ ) and soluble DPP- $4^{24}$; another study reported that it also upregulates expression of adiponectin, which causes an anti-inflammatory effect, ${ }^{25}$ indicating that DPP-4 may exert many functions, both positive and negative, directly and indirectly, on cardiovascular districts.

There were 2 animal studies which supported the role of DPP-4 in atherosclerosis among females. One showed that DPP-4 plays a role in the development of Westerndiet-induced aortic stiffening, vascular oxidative stress, endothelial dysfunction, and vascular remodeling in female mice. ${ }^{26}$ Another study, by inhibiting DPP-4, reduced Western-diet-induced, myocardial-cardiac-restricted overexpression of TRAF3-interacting protein 2 and further induced myocardial inflammation and fibrosis in female mice. $^{27}$

We did not find an association of dyslipidemia or other traditional risk factors in the gene variants we studied. Further studies should be conducted in order to verify this association. However, the available literature verifies that the $D P P-4$ gene is highly polymorphic among different populations and that some $D P P-4$ loci are related to higher plasma lipid levels and cardiovascular risk.

Another study demonstrated that DPP-4 variants in Chinese type 2 diabetes patients were associated with lipid levels. Patients with the rs7608798 SNP exhibited significantly lower serum TG levels in our study and, in the subgroup of women, a lower risk of CAD. For rs3788979, serum TG levels were significantly different among genotypes in our study, and the subgroup of women had a higher risk of CAD. Thus, some women with CAD may have higher lipid levels and cardiovascular risk due to their $D P P-4$ polymorphisms. On the other hand, some women may have lower lipid levels and cardiovascular risk thanks to their $D P P-4$ polymorphisms.

This study had several limitations. Firstly, we only investigated 2 genetic variants for each gene, so we cannot exclude the possibility that other polymorphisms are 
associated with CAD risk. Another limitation of this study is the lack of a complete plasma lipid panel and allowing the use of statins. Furthermore, the control group consisted of patients with symptoms of CAD and negative coronarography findings, but microvessel disease cannot be excluded. It is unknown how the DPP-4 genetic variants affect the function of DPP-4. The biological mechanisms on how DPP-4 influences CAD have not been thoroughly elucidated, although some mechanism has been hypothesized in several animal and human studies. Because of our relatively small sample size, multiple independent studies with larger sample populations are required to validate our findings.

\section{Conclusions}

Our study is the first to demonstrate that CAD pathogenesis is influenced by gender differences in polymorphisms of the DPP-4 gene. This study provides new information on the association of DPP-4 polymorphisms with the risk of CAD in the Taiwanese population. Further studies should be performed to verify this association.

\section{ORCID iDs}

Shih-Min Chiang (1) https://orcid.org/0000-0003-3306-4927 Kwo-Chang Ueng (1) https://orcid.org/0000-0002-5136-7326 Yi-Sun Yang (10) https://orcid.org/0000-0002-6004-7607

\section{Reference}

1. Joseph $\mathrm{P}$, Leong D, McKee M, et al. Reducing the global burden of cardiovascular disease. Part 1: The epidemiology and risk factors. Circ Res. 2017;121(6):677-694.

2. Leong DP, Joseph PG, McKee M, et al. Reducing the global burden of cardiovascular disease. Part 2: Prevention and treatment of cardiovascular disease. Circ Res. 2017;121(6):695-710.

3. Lopez AD, Mathers CD, Ezzati M, Jamison DT, Murray CLJ. Global and regional burden of disease and risk factors, 2001: Systematic analysis of population health data. Lancet. 2006;367(9524):1747-1757.

4. Wang B, Fu ZY, Ma YT, et al. Identification of a CYP19 gene singlenucleotide polymorphism associated with a reduced risk of coronary heart disease. Genet Test Mol Biomarkers. 2016;20(1):2-10.

5. Winkelmann BR, Hager J. Genetic variation in coronary heart disease and myocardial infarction: Methodological overview and clinical evidence. Pharmacogenomics. 2000;1(1):73-94.

6. Inzucchi SE, McGuire DK. New drugs for the treatment of diabetes. Part II: Incretin-based therapy and beyond. Circulation. 2008;117(4): 574-584.

7. Augustyns K, Bal G, Thonus G, et al. The unique properties of dipeptidyl-peptidase IV (DPPIV/CD26) and the therapeutic potential of DPP IV inhibitors. Curr Med Chem. 1999;6(4):311-327.
8. Duan L, Rao X, Xia C, et al. The regulatory role of DPP-4 in atherosclerotic disease. Cardiovasc Diabetol. 2017;16:76.

9. Pala L, Rotella CM. The role of DPP-4 activity in cardiovascular districts: In vivo and in vitro evidence. J Diabetes Res. 2013;2013:590456.

10. Kazafeos K. Incretin effect: GLP-1, GIP, DPP-4. Diabetes Res Clin Pract. 2011;93(Suppl 1):S32-S36.

11. White WB, Cannon CP, Heller SR, et al; EXAMINE Investigators. Alogliptin after acute coronary syndrome in patients with type 2 diabetes. N Engl J Med. 2013;369(14):1327-1335.

12. Scirica BM, Bhatt DL, Braunwald E, et al; SAVOR-TIMI 53 Steering Committee and Investigators; Saxagliptin and cardiovascular outcomes in patients with type 2 diabetes mellitus. N Engl J Med. 2013;369: 1317-1326.

13. Green JB, Bethel MA, Armstrong PW, et al; TECOS Study Group. Effect of sitagliptin on cardiovascular outcomes in type 2 diabetes. NEngl J Med. 2015;373(3):232-242.

14. Xing X, Han Y, Zhou X, et al. Association between DPP-4 gene polymorphism and serum lipid levels in Chinese type 2 diabetes individuals. Neuropeptides. 2016;60:1-6.

15. AghiliN, Devaney JM, Alderman LO, Zukowska Z, Epstein SE, BurnettMS. Polymorphisms in dipeptidyl peptidase IV gene are associated with the risk of myocardial infarction in patients with atherosclerosis. Neuropeptides. 2012;46(6):367-371.

16. Spence JD, Pilote L. Importance of sex and gender in atherosclerosis and cardiovascular disease. Atherosclerosis. 2015;241(1):208-210.

17. Orth-Gomer K, Deter HC. Sex and gender issues in cardiovascular research. Psychosom Med. 2015;77(9):1067-1068.

18. Hausenloy DJ, Whittington HJ, Wynne AM, et al. Dipeptidyl peptidase-4 inhibitors and GLP-1 reduce myocardial infarct size in a glucose-dependent manner. Cardiovasc Diabetol. 2013;12:154.

19. Chinda K, Palee S, Surinkaew S, Phornphutkul M, Chattipakorn S, Chattipakorn N. Cardioprotective effect of dipeptidyl peptidase-4 inhibitor during ischemia-reperfusion injury. Int J Cardiol. 2013;167(2): 451-457.

20. Silva Jr WS, Godoy-Matos AF, Kraemer-Aguiar LG. Dipeptidyl peptidase 4: A new link between diabetes mellitus and atherosclerosis? Biomed Res Int. 2015;2015:816164.

21. Sato Y, KoshiokaS, Kirino Y, et al. Role of dipeptidyl peptidase IV (DPP-4) in the development of dyslipidemia: DPP-4 contributes to the steroid metabolism pathway. Life Sci. 2011;88(1-2):43-49.

22. Lei $Y$, Yang G, Hu L, et al; FAHA. Increased dipeptidyl peptidase-4 accelerates diet-related vascular aging and atherosclerosis in ApoEdeficient mice under chronic stress. Int J Cardiol. 2017;243:413-420.

23. Mulvihill EE, Varin EM, Ussher JR, et al. Inhibition of dipeptidyl peptidase-4 impairs ventricular function and promotes cardiac fibrosis in high fat-fed diabetic mice. Diabetes. 2016;65(3):742-754.

24. Shah Z, Kampfrath T, Deiuliis JA, et al. Long-term dipeptidyl-peptidase 4 inhibition reduces atherosclerosis and inflammation via effects on monocyte recruitment and chemotaxis. Circulation. 2011; 124(21):2338-2349.

25. Ida S, Murata K, Betou K, et al. Effect of trelagliptin on vascular endothelial functions and serum adiponectin level in patients with type 2 diabetes: A preliminary single-arm prospective pilot study. Cardiovasc Diabetol. 2016;15:153.

26. Manrique C, Habibi J, Aroor AR, et al. Dipeptidyl peptidase-4 inhibition with linagliptin prevents Western diet-induced vascular abnormalities in female mice. Cardiovasc Diabetol. 2016;15:94.

27. Aroor AR, Habibi J, Kandikattu HK, et al. Dipeptidyl peptidase-4 (DPP-4) inhibition with linagliptin reduces Western diet-induced myocardial TRAF3IP2 expression, inflammation and fibrosis in female mice. Cardiovasc Diabetol. 2017;16(1):61. 